\title{
Our Experience of Surgical Treatment of Cicatrical Stenosis of the Trachea
}

\author{
Rustem Hayaliev*, Shuhrat Khudaybergenov and Otabek Eshonkhodjaev \\ Associate Professor, Thoracic Surgeon, Candidate of Medical Sciences, Uzbekistan
}

*Corresponding author: Hayaliev Rustem Yakubovich, Associate Professor, Thoracic Surgeon, Candidate of Medical Sciences, Uzbekistan

\begin{tabular}{|c|c|}
\hline ARTICLE INFO & ABSTRACT \\
\hline 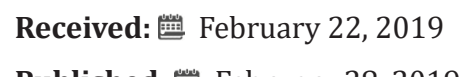 & $\begin{array}{l}\text { Citation: Rustem H, Shuhrat K, Otabek E. Our Experience of Surgical Treatment of Cicatrical } \\
\text { Stenosis of the Trachea. Biomed J Sci \& Tech Res 15(3)-2019. BJSTR. MS.ID.002690. }\end{array}$ \\
\hline
\end{tabular}

\section{Relevance of the Problem}

The Development and achievements of modern anesthesiology and reanimatology make it possible to save patients who were previously considered incurable and hopeless. In turn, prolonged mechanical ventilation through intubation or tracheostomy tubes is the main cause of damage to the tracheal wall with the formation of tracheal stenoses. At the same time, the number of patients with this pathology in recent years does not tend to decrease. Severe complications, possible after resection of the trachea, such as the failure of the anastomotic suture line, bleeding, mediastinitis, restenosis, paresis of the larynx, continue to occur. Thus, according to various authors, their frequency reaches $25 \%$. The mortality rate remains high - up to $8 \%$. The treatment of long and multifocal stenoses of the respiratory tract, when different parts of the trachea, as well as the combined defeat of the trachea and larynx are involved in the process, remains a difficult and not fully resolved problem. Stenosis of the upper respiratory tract is one of the main causes of long-term disability and invalidity of patients, leading to social and professional maladjustment. In this regard, the problem of narrowing of the trachea from medical develops into socialeconomic, as the main contingent of this category of patients are young and able-bodied people.

Another relevant issue is the determination of indications and contraindications for resection of the trachea in patients with multifocal lesions of the trachea (Samokhin AY (1992); Perevoznikova IA, Kozak AR (2010); Abbasiezfouli A, Shadmehr MB (2007). According to the literature, some authors recommend to maximize the use of the resection methods of treatment [Wright CD, Grillo HC, Wain JC (2004)]. Despite this, taking into account the peculiarities of extended and multifocal tracheal stenoses, in most cases, the optimal method of treatmentis step-by-step reconstructive plastic surgery. The final stage of surgical rehabilitation of patients with chronic stenosis of the larynx and trachea is plastic closure of persistent laryngotracheal defect. Closure of stoma can be performed only when a stable epithelialized adequate lumen of the respiratory tract is formed, and the possibility of restenosis is excluded.

The aim of the study was to improve the results of surgical treatment of patients with stenosis and persistent extensive defects of the anterior tracheal wall.

\section{Materials and Methods}

Analysis of treatment results of 141 patients with stenosis of the trachea and larynx, from 2008 to 2018.92 patients underwent reconstructive operations in the form of plasty trachea, including incision of the stenosis, excision of scar tissue and the formation of the lumen of the T-shaped endoprosthesis. The period of dilation on a T-shaped endoprosthesis varies from 7 to 14 months, depending on individual parameters in the formation of a wide persistent tracheal lumen with an epithelized surface without signs of recurrence of stenosis. In 7 (4.9\%) patients after removal of T-shaped endoprosthesis in the period of control observation after 7-10 days there was a relapse of stenosis in the projection below the tracheostomy. In such cases, patients underwent repeated operation with the formation of a lumen on a T-shaped endoprosthesis with a good long-term result. In $7(4,9 \%)$ patients after decannulation (removal of the T-shaped endoprosthesis) at the time of 7-14 days narrowing of the trachea due to the lack of 
frame-based structures (due to tracheomalacia), and the growth of granulation was marked, they underwent single-step circular resection of the trachea with anastomosis end-to-end and the elimination of the defect of the anterior wall of the trachea and soft tissues of the neck. Primary circular resection of trachea (CRT) was performed in 32 cases.

\section{The Results of the Study}

Patients ( $n=92)$ with post tracheostomic defects of the anterior wall of the trachea and soft tissues of the size from $1.0 \mathrm{~cm}$ to $2.0 \mathrm{~cm}$ wide and $2.0 \mathrm{~cm}$ to $4.0 \mathrm{~cm}$ in height, while the depth of the defect more than $8 \mathrm{~mm}$, i.e. the anterior-posterior size of the lumen of the trachea and persistent formed the lumen of the airway in the pathologic area without the presence of the protector, thelocal threelayer or four-layer skin-muscle plasty of the defect were performed Ten patients with extensive defects of the anterior-lateral walls of the trachea and distal larynx underwent reconstructive surgery using microsurgical techniques. It should be noted that these patients with extensive defects of the anterior wall of the trachea had previously undergone other stages of treatment: installation of a T-shaped stent - 4 cases; 3 patients with skin-muscle plastic surgery by Bokstein and 3 cases of autorib cartilage implantation. Thus, in all 10 cases, local tissues were not enough to close the defect due to the large sizes. This required the use of microsurgical techniques to move autorib implants, the supraclavicular skin and fascial flap and, if necessary, split thigh skin. Performing circular tracheal resection $(n=39)$ allows to radically cure the patient in one stage with good immediate and long-term results, in the absence of contraindications from concomitant diseases it can be performed at the first stage of treatment.

In the case of contraindications, it is possible to perform CRT at the next stage after endoscopic and/or staged endosurgical treatment in the case of restenosis of the lumen. The length of resected tracheal sections ranged from 1.8 to $6 \mathrm{~cm}$. In 1 case in a patient admitted from another medical hospital with cicatricial stenosis of the anastomosis of the trachea performed reresection of the trachea. CRT was complicated by the failure of anastomosis in the early postoperative period in 2 patients (1.4\%). Of these, in one case, the patient was saved by performing an emergency operation, pulling the severed and contracted distal and proximal ends of the anastomosis into the neck wound and forming a tracheal lumen on the T-shaped stent. In the second case, the failure of the sutures as a result of excessive tension of the anastomosis after extended resection led to the separation of the anastomosis, complicated by erosive bleeding and fatal outcome. In other cases, after CRT we did not observed stenosis of anastomosis and recurrent laryngeal nerve paresis. Control endoscopic examinations were performed on the 5th day after the operation. Then in 1 month, 3 months, 6 months and a year after surgery. In all cases, good immediate and long-term results were obtained. Long-term results of treatment were evaluated and studied in terms of 6 months to 7 years in 129 (91.4\%) patients. In 10 patients, one of the stages of complex treatment continues, the patients are under dynamic endoscopic control.

\section{Conclusion}

Reconstruction of the T-shaped endoprosthesis in patients with tracheolaringeal and extended cicatrical stenoses of trachea allows to rehabilitate patients with heavy concomitant pathology, to eliminate the signs of violation of the sputum of the bronchi, to preserve the possibility of breathing through the natural airway and restore phonation, eliminating the risk of migration and obstruction of the prosthesis and allows to create stable and adequate lumen of the trachea with the subsequent performance of plastic operations for the closure of the defect. In patients with multifocal and multilevel tracheal stenosis in the presence of neurological deficit and severe concomitant pathology, when performing CRT is impossible, reconstruction on T-shaped endoprosthesis is the "Gold Standard". With extended scar stenosis of the trachea involving more than $30 \%$ of the trachea, up to $5-6 \mathrm{sm}$, it is possible to perform extensive CRT under conditions of sufficient mobilization at the necessary levels to eliminate tension in the area of the created anastomosis. In the absence of contraindications from concomitant diseases, CRT can be performed at the first stage of treatment. In the case of contraindications, it is possible to perform CRT at the next stage of treatment.

\section{ISSN: 2574-1241}

DOI: 10.26717/BJSTR.2019.15.002690

Hayaliev Rustem Yakubovich. Biomed J Sci \& Tech Res

This work is licensed under Creative Commons Attribution 4.0 License

Submission Link: https://biomedres.us/submit-manuscript.php

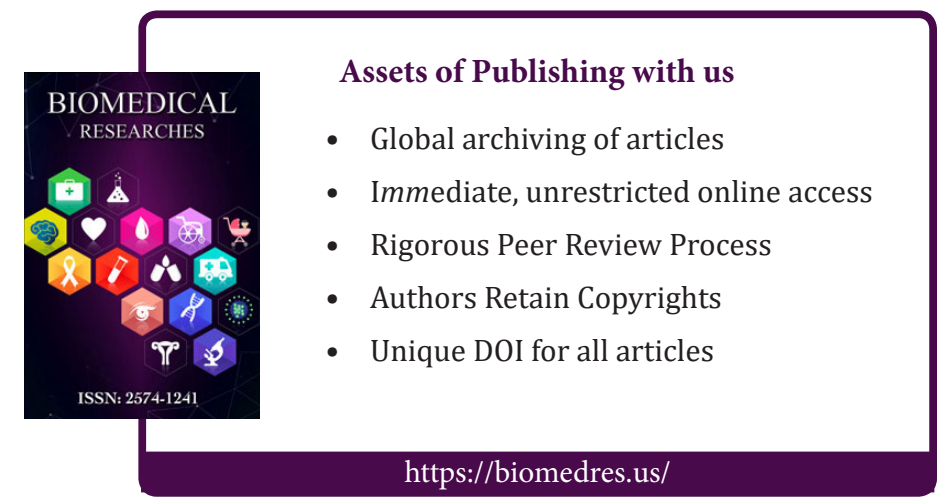

